\title{
Technical Note: Wet validation of optical tomography for drinking water discolouration studies
}

\author{
R. Floris, P. van Thienen, and H. Beverloo \\ KWR Watercycle Research Institute, P.O. Box 1072, 3430 BB Nieuwegein, the Netherlands \\ Correspondence to: R. Floris (roberto.floris@kwrwater.nl) \\ Received: 21 December 2012 - Published in Drink. Water Eng. Sci. Discuss.: 11 February 2013 \\ Revised: 16 May 2013 - Accepted: 30 May 2013 - Published: 13 June 2013
}

\begin{abstract}
This paper presents a set of validation experiments for the reconstruction of a cross-sectional particle concentration field in a transparent pipe filled with a fluid using optical tomography.
\end{abstract}

\section{Introduction}

Discoloured water still represents the second most common reason (after not getting any water) of customers' complaints to water companies (Vreeburg and Boxall, 2007; Husband and Boxall, 2011).

The origin of discoloured water is correlated with the presence of waterborne particles into the drinking water distribution system for which fate and behaviour are to a large degree controlled by the flow conditions (Husband et al., 2008). Changes in the flow velocity modify the magnitude of the forces acting upon the particles and as a consequence affect the radial transport mechanisms leading to deposition or resuspension. Theoretical investigations were conducted in order to understand the relation between flow conditions and particle transport mechanisms and how they affect the crosssectional particle distribution (van Thienen et al., 2011b). An optical tomography system was developed and tested for the first time in drinking water research towards an experimental verification of these theoretical findings (van Thienen et al., 2011a). More specifically, it was aimed at the detection of the transition from turbulent diffusion dominated radial transport to turbophoresis dominated radial particle transport. The former mechanisms works to homogenize the particle concentration field, whereas the latter drives particles towards the wall. These particle configurations were recreated in the lab for the validation of the tomography system (see Fig. 1).

Tomography is a non-invasive technique of imaging that allows the study of the internal structures of an object or a system without influencing or conditioning it. The common basic principle of these techniques is to analyze the interac- tion between the system studied and a physical field and then to translate this information into images of the system under study.

The optical tomography technique was used here to reconstruct cross-sectional particle concentration fields in a transparent pipe using the interaction between light and particles suspended in a fluid. van Thienen et al. (2011a) give a full description of the applied methodology, including synthetic tests of the mathematical procedure and dry tests of the actual device, using wire mesh curtains to represent particle clouds in the measurement domain. Following their recommendation, a more complex optical model was implemented in the inversion procedure, which is described below.

Although the previous results of van Thienen et al. (2011a) showed the validity of the mathematical framework and capability of the optical tomography system in resolving semitransparent objects, test conditions which are more representative of the actual intended application of the device are necessary. Because cross-sectional particle distributions in flowing water in a pipe are difficult to control, wet quasi-static tests in a highly viscous fluid were performed in order to asses the value of the tomograms obtained from measurements. Here we present the validation of the system distinguishing different particle clouds patterns expected by the theoretical model. Also, a suitable value for the system variance as used in the computation of the tomograms was determined from the observations. 

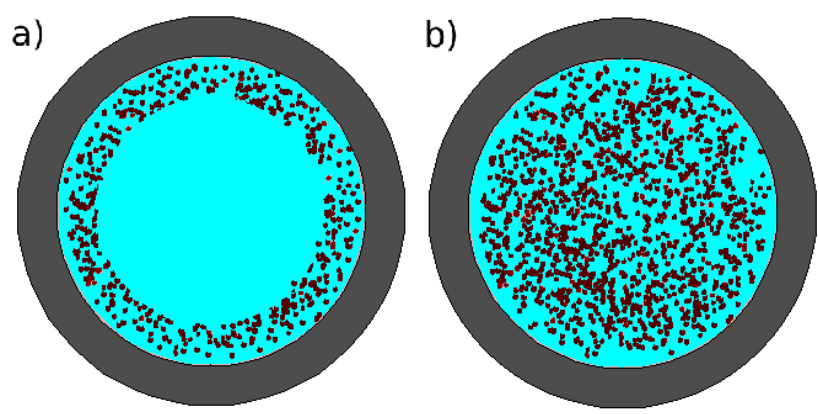

Figure 1. A schematic representation of the two model patterns created for the validation of the tomography system. (a) centered full ring pattern $1 \mathrm{~cm}$ thick; (b) homogeneous particle distribution.

\section{Methods}

\subsection{Optical tomography}

van Thienen et al. (2011a) give a complete description of the methodology and optical tomography measuring device (OTD), which is briefly summarized here. The OTD consists of a PVC ring containing, evenly spaced and alternatingly, 10 high power light emitting diodes (LED) and 10 light sensors (LS). The LEDs and the LSs are controlled by an Arduino board (Arduino, 2010) on which is installed custom made control software. The PVC ring is mounted on a transparent section of pipe which is part of a lab test rig, in which hydraulic conditions relevant for drinking water distribution can be simulated. The device generates light pulses using the LEDs and detects the result of the interaction between these pulses and the suspension using the light sensors. The LEDs are operated sequentially and for every light pulse data are collected by every LS. The data collected are transmitted to the workstation which computes a tomogram of the light absorption coefficient field with an inverse problem algorithm (van Thienen et al., 2011a). The tomogram is a twodimensional image representing the variation of the light absorption coefficient representative of the local particle concentration through a cross-section of the suspension flow.

The mathematical framework of the tomography is based on work by Tarantola (2005) and Muntendam-Bos et al. (2008) and revolves around the following equation:

$\boldsymbol{s}=\mathbf{C}_{\mathrm{m}} \mathbf{A}^{T}\left(\mathbf{A C}_{\mathrm{m}} \mathbf{A}^{T}+\mathbf{C}_{\mathrm{d}}\right)^{-1} \boldsymbol{r}$

In this expression, vector $\boldsymbol{r}$ contains all measurements, vector $s$ has all unknown absorption coefficients and matrix A contains all integration coefficients. $\mathbf{C}_{\mathrm{m}}$ is the covariance matrix of the prior model matrix and $\mathbf{C}_{\mathrm{d}}$ is the prior data covariance matrix, which has the variances $\sigma^{2}$ of the measurements on the main diagonal and zeros off the main diagonal. For a more detailed description, the reader is referred to van Thienen et al. (2011a). The total variances consist of the actual measurement variances $\sigma_{m i j}^{2}$ which are obtained for each LED-sensor combination $i j$ from multiple samples and a sys-

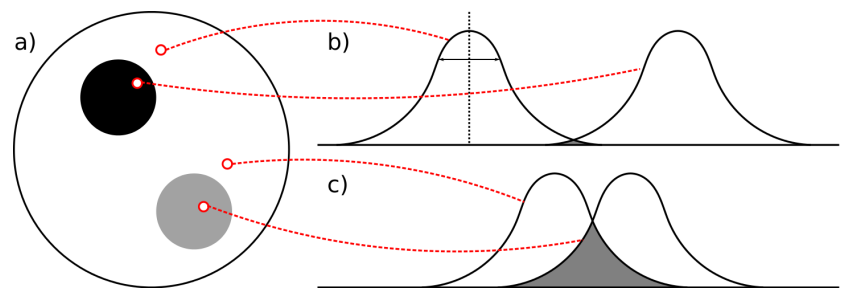

Figure 2. Tomogram showing two distinct circular features (a). The first of these is very different from the background, resulting in a small overlap (b) of the associated probability distributions for the two sampled points. The bottom feature is much closer to the background and has a larger overlap (c) of the associated probability distributions for the two sampled points.

tem variance $\sigma_{\mathrm{s}}^{2}$, which are summed following Bienaym's formula:

$\sigma_{i j}^{2}=\sigma_{m i j}^{2}+\sigma_{\mathrm{s}}^{2}$

Using this mathematical framework, a mathematical solution can always be found for the inversion problem (i.e. the reconstruction of the cross-sectional particle concentration field from the light measurements). The question, however, is to what extent the obtained image represents physical features instead of numerical artifacts and noise. The applied method not only computes a model for the physical configuration, but also the corresponding posterior variances for the model values at all nodes. When a feature is observed in a tomogram, the variances can answer the question whether the feature is statistically significant, i.e. whether it is really different from the background. A small overlap of the probability density functions (Fig. 2a, b) signifies a low likelyhood of the values actually being the same, whereas a large overlap (Fig. 2a, c) suggests a high probability. By choosing a confidence level, e.g. $95 \%$, a maximum amount of overlap for which values are considered different is obtained, in this case 0.05 .

The main parameters affecting the width of the probability distributions are the different components of the prior data variance (van Thienen et al., 2011a). These are the measurement component $\sigma_{\mathrm{m}}$ and the system component $\sigma_{\mathrm{s}}$. The former parameter is computed from a series of measurements and represents the noise in the measurements. All other errors and uncertainties in the system, i.e. in the discretization, systematic errors in measurement and analog-digital conversion, etc. are included in the latter. This parameter is difficult to determine exactly. Therefore, it should be estimated on the basis of validation experiments such as those presented in this paper. In these validation experiments, the physical structure which is reconstructed in the tomogram is known, so the appropriate value for $\sigma_{\mathrm{s}}$ can be selected which results in an accurate reconstruction without artefacts. 


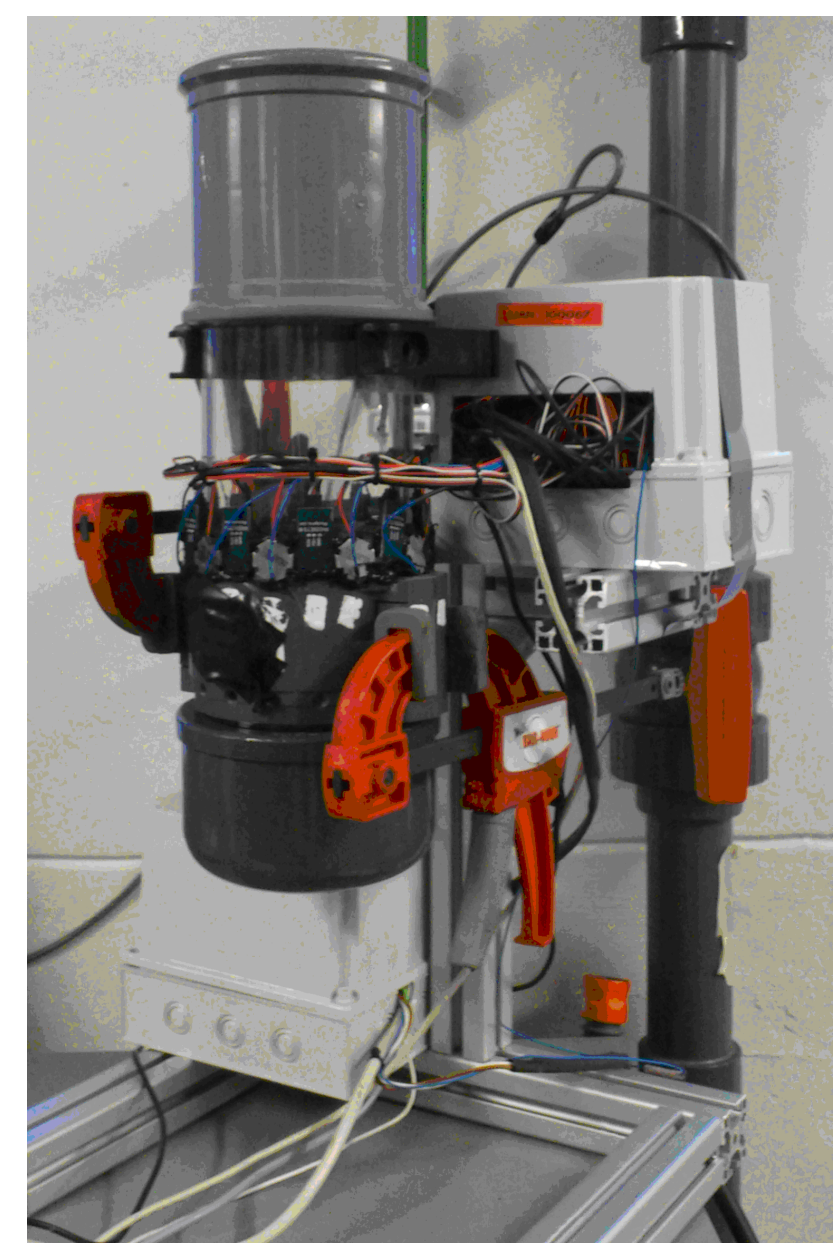

Figure 3. Mounting of the optical tomograohy device on a vertical end of transparent pipe.

\subsubsection{Enhancement of the optical model}

The optical model was improved relative to the approach described in van Thienen et al. (2011a). Originally, it was assumed that particles are never in the shadows of other particles, which means that the light intensity decreases linearly with the number of particles encountered. This is a reasonable approximation for low particle concentrations, but not for the higher concentrations which are applied in this work. Here, we apply an exponential extinction model based on the Lambert-Beer law:

$\mathrm{d} I=-\frac{\sigma N}{\mathrm{~d} x}$

with $I$ the light intensity, $\sigma$ the absorption cross section and $N$ the number density of absorbers, assuming a linear variation of the particle concentration between model nodes.

\subsubsection{Experimental set-up}

The wet quasi-static tests were performed mounting the Optical Tomography Device on a vertical transparent tube containing a glycerol solution ( $80 \%$ in volume), see Fig. 3. Used coffee granules (i.e. the leftovers after making coffee) with a particle size of 500-600 $\mu \mathrm{m}$ were chosen as a testing material because of three factors: their density (900$1100 \mathrm{~kg} \mathrm{~m}^{-3}$ ), which is close to the density of sediment samples collected from DWDSs (Boxall et al., 2001) their optical properties (high light absorption and low light reflection) and their physical and chemical stability after extraction (the soluble substances have already been extracted in the process of making coffee). Particles were left in the glycerol solution overnight before the experiments in order to obtain a stable dispersion at high concentration $\left(400 \mathrm{~g} \mathrm{~L}^{-1}\right)$. The OTD was placed far from the free surface of the liquid $(15 \mathrm{~cm})$ in order to avoid artefacts originating from light reflections at this interface. Two model cross-sectional particle concentration distributions (a centered full ring pattern $1 \mathrm{~cm}$ thick and homogeneous pattern) were created injecting the high concentrated coffee dispersion into the clear glycerol solution using a syringe. A schematic representation of the two patterns is given in Fig. 1. Complete screening of the experimental setup from external light sources was ensured during the measurements.

\section{Results}

Tomograms for a single selected representative frame for each of the two experimental configurations described above are presented in Fig. 4. These show tomograms for four different values of the system error $\sigma_{\mathrm{s}}(0.08,0.12,0.15$ and 0.20 ). Also included are probability density distributions for four selected nodes and their respective amounts of overlap.

\section{Discussion}

As can be seen in Fig. 4, both the ring structure and the homogeneous structure can be clearly resolved when an appropriate value of $\sigma_{\mathrm{s}}$ is chosen. For the ring structure to be statistically significant in the tomogram, i.e. for the outside part of the tomogram to be significantly different ( $95 \%$ confidence interval) from the inside, we find the requirement that $\sigma_{\mathrm{s}} \leq 0.15$. For the homogeneous structure to be statistically significant in the tomogram, i.e. for the outside part of the tomogram to be not significantly different ( $95 \%$ confidence interval) from the inside, the requirement is found that $\sigma_{\mathrm{s}} \geq 0.12$. Therefore, there is a range of $\sigma_{\mathrm{s}}$ between 0.12 and 0.15 where a statistically significant distinction can be made between ring structures and homogeneous structures for the applied particle concentration. The particle concentration which was used is $400 \mathrm{~g} \mathrm{~L}^{-1}$. Some local mixing after injection resulted in a slightly lower effective particle concentration. When this value is also strived for in dynamic 


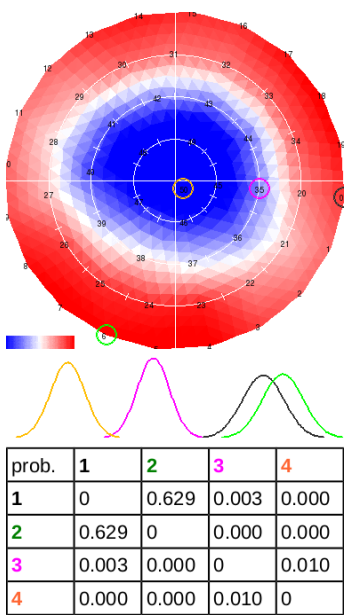

(A) $\sigma_{s}=0.08$

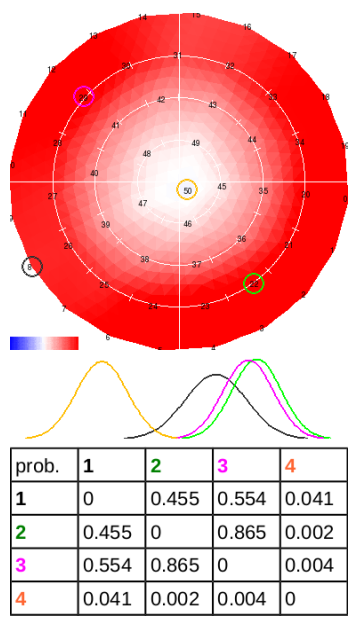

(E) $\sigma_{s}=0.08$

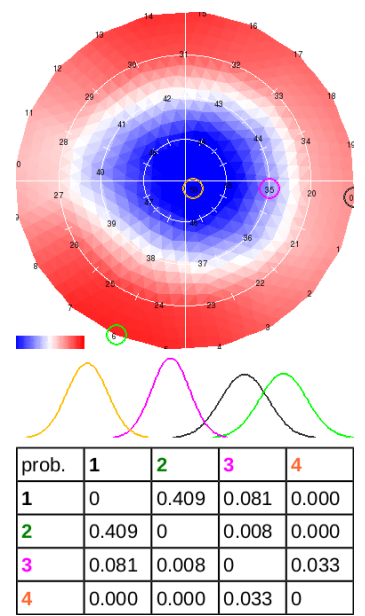

(B) $\sigma_{s}=0.012$

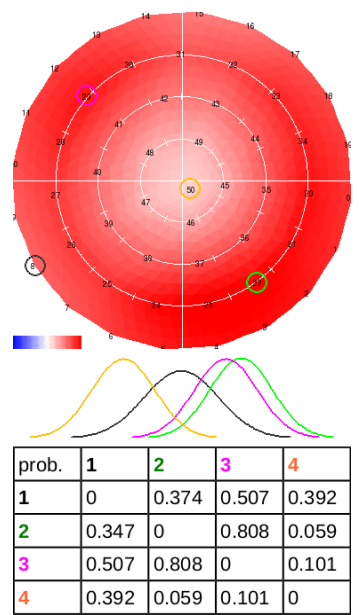

(F) $\sigma_{s}=0.12$

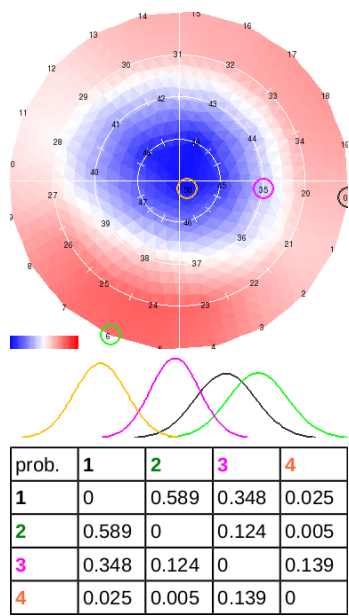

(C) $\sigma_{s}=0.015$

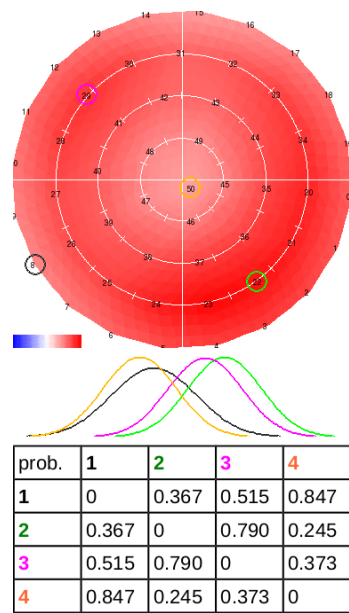

(G) $\sigma_{s}=0.15$

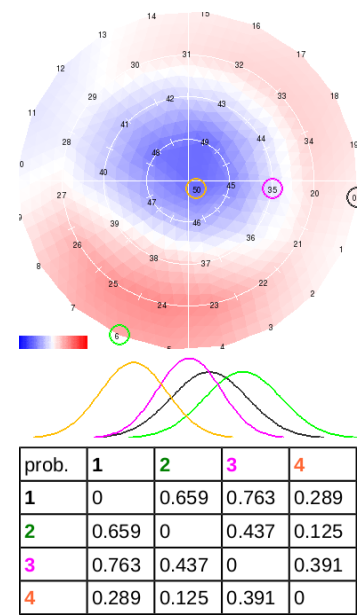

(D) $\sigma_{s}=0.20$

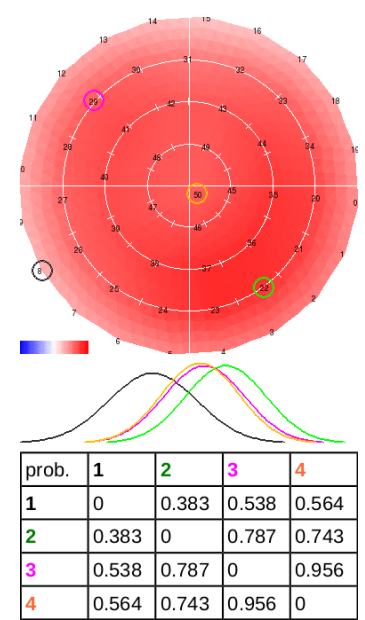

(H) $\sigma_{s}=0.20$

Figure 4. Tomograms, posterior probability density functions and associated amounts of overlap for selected nodes in the tomograms. Selected node markers and associated curves are marked in the same colour in each case. Both a ring structure: (A-D) and a homogeneous case (E-H) are displayed.

experiments (in contrast to the current quasi-static) in which the optical tomograph is applied, the same resolvability of the ring and homogeneous structures is expected. Finally, when applying the insights into statistical significance presented in the technical note to the results of the dry validation experiments of the optical tomograph in van Thienen et al. (2011a), we find that the observed linear and annular structures in that work are also statistically significant.

\section{Conclusions}

The optical tomography setup described in van Thienen et al. (2011a) is capable of distinguishing (95\% confidence interval) between ring shaped and homogeneous particle concentration fields in transparent pipe sections sampled by the tomograph at particle concentrations of $400 \mathrm{~g} \mathrm{~L}^{-1}$ when assuming a system standard deviation $0.12 \leq \sigma_{\mathrm{s}} \leq 0.15$. Therefore, it may be applied to experimentally validate location of the transition between turbulent diffusion and turbophoresis dominated radial particle transport predicted from theory by van Thienen et al. (2011b).

Edited by: J. Boxall

\section{References}

Arduino: http://www.arduino.cc, last access: 6 December 2010. Boxall, J. B., Skipworth, P. J., and Saul, A. J.: A novel approach to describing sediment movement in distribution mains, based on measured particle characteristics, Proc. International CCWI conference, 3-5 September 2001, De Montfort University, UK, 2001. 
Husband, P. S. and Boxall, J. B.: Asset deterioration and discolouration in water distribution systems, Water Res., 45, 113-124, 2011.

Husband, P. S., Boxall, J. B., and Saul, A.: Laboratory studies investigating the processes leading to discolouration in water distribution networks, Water Res., 42, 4309-4318, 2008.

Muntendam-Bos, A. G., Kroon, I. C., and Fokker, P. A.: Time-dependent Inversion of Surface Subsidence due to Dynamic Reservoir Compaction, Math. Geosci., 40, 159-177, doi:10.1007/s11004-007-9135-3, 2008.

Tarantola, A.: Inverse Problem Theory and Methods for Model Parameter Estimation, Society for Industrial and Applied Mathematics, Philadelphia, 2005. van Thienen, P., Floris, R., and Meijering, S.: Application of optical tomography in the study of discolouration in drinking water distribution systems, Drink. Water Eng. Sci., 4, 61-69, doi:10.5194/dwes-4-61-2011, 2011a.

van Thienen, P., Vreeburg, J., and Blokker, E.: Radial transport processes as a precursor to particle deposition in drinking water distribution systems, Water Res., 45, 1807-1818, 2011 b.

Vreeburg, J. and Boxall, J.: Discolouration in potable water distribution systems: A review, Water Res., 41, 519-529, 2007. 